METALLOENZYMES

Copper chews on cellulose

Proc. Natl. Acad. Sci. USA 108, 15079-15084 (2011)

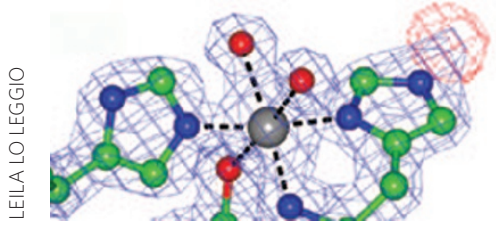

Degradation of cellulose-rich biomass is necessary for its conversion to a variety of commercially relevant end products. Previous work identified enzymes from glycoside hydrolase family GH61 as capable of assisting cellulases in breaking down cellulose's complex structure. However, the way in which the GH61 enzymes contributed to this process was unknown, as the available structures (which lack canonical active site residues and sugar-binding pockets) and their requirement for a metal are inconsistent with glycoside hydrolase activity. Quinlan et al. now shed light on this question in their exploration of a GH61 from Thermoascus aurantiacus (TaGH61). Hydrolysis assays confirmed that TaGH61's function is independent of and complementary to that of cellulases and requires a redox-active cofactor. Characterization of TaGH61's products further indicated that the enzyme oxidatively depolymerizes the cellulose chains, thus exposing new sites for hydrolysis by cellulases. The unknown metal was identified by ITC and EPR spectroscopy as copper, bound with a $K_{\mathrm{D}}<1 \mathrm{pM}$ and with tetragonal coordination geometry. Finally, an X-ray structure showed
$\mathrm{Cu}(\mathrm{II})$ binding reminiscent of copper methane monooxygenase, suggesting that TaGH61 uses oxygen activation as a strong thermodynamic driving force for cellulose breakdown. Surprisingly, the structure also revealed a methyl group on one of the coordinating histidine ligands. As this modification is unprecedented for metalloenzymes, further investigation will be required to understand the impact of this methyl group on metal reactivity.

\section{DRUG DISCOVERY}

\section{Sidelining c-Myc Cell 146, 904-917 (2011)}

Overexpression of the transcriptional activator c-Myc is associated with cell proliferation in numerous malignancies. However, direct pharmacological inhibition of c-Myc has proven difficult. Delmore et al. now show that c-Myc activity can be downregulated in cancer cell lines through an indirect route involving the perturbation of coactivator protein recruitment to chromatin. Using expression analysis and RNA interference, the authors showed that BRD4, a member of the bromodomain and extraterminal (BET) class of transcriptional coactivators, is associated with disease progression in multiple myeloma (MM). The authors hypothesized that JQ1, a triazolodiazepine compound that specifically inhibits BET protein binding to acetyllysinemodified histones in chromatin, could be used as a chemical probe to explore the link between BET proteins and c-Myc regulation in MM. Treatment of MM cells with JQ1 disrupted the transcriptional programs downstream of c-Myc. Surprisingly, JQ1

\section{STEM CELLS}

\section{Oxidative renewal}

Reactive oxygen species (ROS) and DNA repair defects are known to limit the regenerative potential of hematopoietic stem cells (HSCs), which is necessary to replenish mature blood cells. Yahata et al. now provide evidence that these two insults to HSCs are related: long-term production of ROS leads to the accumulation of DNA damage and exhausts HSCs. The authors demonstrated that human HSCs serially transplanted into mice gradually lost their proliferative capacity and accumulated marks of DNA damage, such as $\gamma-H 2 A X$ foci. ROS production increased with each serial transplant, and ROS high cells lost their repopulation potential by the third transplant. HSCs with elevated ROS levelsinduced by inhibition of glutathione synthetase with buthionine sulfoximine (BSO)-had increased numbers of $\gamma-\mathrm{H} 2 \mathrm{AX}$ foci, expressed DNA damage-response proteins and showed diminished repopulating capacity. The authors further demonstrated that HSCs are more sensitive to ROS production than progenitor cells from the same lineage, showing that inhibition with BSO yielded comparable amounts of ROS in both cell types. The HSCs, however, showed more cell-cycle arrest as well as faster and more persistent accumulation of $\gamma-\mathrm{H} 2 \mathrm{AX}$ foci. Inhibition of ROS production with an antioxidant blocked the accumulation of BSO-induced DNA damage and improved the self-renewal capacity of HSCs. Taken together, these data indicate that ROS play a causative role in the accumulation of DNA damage in HSCs and have implications for the renewal capacity of these cells. treatment also blocked transcription of $M Y C$ itself in a dose-dependent manner. Chromatin immunoprecipitation revealed that BRD4 accumulates at immunoglobulin enhancer elements abnormally fused to the MYC gene in MM and that JQ1 can deplete these strong enhancers of BRD4. These results suggested that pharmacological inhibition of BETchromatin interactions has the potential to block $M Y C$-induced cell proliferation-a prediction that was validated in several mouse models of multiple myeloma.

MALARIA

\section{An addiction to isoprenoids PLoS Biol. 9, e1001138 (2011)}

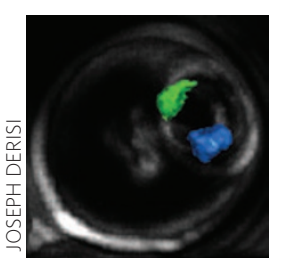

The apicoplast in the malaria-causing Plasmodium spp. is an organelle that is thought to be a remnant of a red algae symbiont. Despite the loss of its photosynthetic capability and most of its genome during evolution, loss of this organelle is lethal during both the liver and blood stages of the parasite life cycle, so it must be inherited at each cell division. Fosmidomycin, known to kill blood-stage parasites, is an inhibitor of the prokaryotic pathway for synthesis of isoprenoid precursors. Yeh et al. have now further explored this biosynthetic pathway as a malarial drug target and found that one of its precursors, isopentenyl pyrophosphate (IPP), was sufficient to rescue the toxic effect of fosmidomycin. Antibiotics such as chloramphenicol or doxycycline that target transcription or translation also block apicoplast gene expression, and this causes a 'delayed-death' phenotype: the treated parasites themselves are not affected, but their progeny lack apicoplasts and ultimately die. IPP could also rescue this phenotype. The parasites live far beyond the first generation after IPP rescue, despite their lack of apicoplasts and apicoplast functions, which include protein import and processing. These results suggest that production of isoprenoid precursors is the only essential function of the malarial apicoplast during the replicative (blood) stage of the parasite, and they highlight the potential of developing drugs against this machinery to target the apicoplast and parasite replication. 